\title{
功能纳米材料研究进展一唐智勇研究员及其团队专访
}

《物理化学学报》编辑部

北京大学化学与分子工程学院, 北京 100871

\section{Interview with Professor Zhiyong Tang and His Research Group: Recent Progress in the Field of Functional Nanomaterials}

\author{
Editorial Office of Acta Physico-Chimica Sinica \\ College of Chemistry and Molecular Engineering, Peking University, Beijing 100871, P. R. China. \\ Email: whxb@pku.edu.cn. \\ Published online: March 24, 2020.
}

功能纳米材料是化学、物理、生物和材料科学多学科交叉的前沿, 是纳米科技发展的基础。功能纳 米材料集中体现了小尺寸、精准控制、高集成度和强相互作用等现代科学技术发展的特点, 是将量子力 学效应工程化或技术化的最好载体之一, 呈现独特的光、电、磁、机械和催化等性质, 在物理、生物、 化学和材料学领域中都有广泛的研究和应用前景。

我们有幸采访了功能纳米材料专家, 《物理化学学报》 “精准纳米合成” 特刊的客座编辑唐智勇研 究员, 为我们分享他在功能纳米材料方面的研究经历及功能纳米材料在国内外的现状、应用、发展方向 等, 并对功能纳米材料领域面临的一些重要问题进行了分析。

问题 1: 唐智勇研究员, 在您的科研生涯中从事过 哪些方面的研究? 您从什么时候开始从事功能纳 米材料方面的研究?

回答: 我从1993年在武汉大学读硕士研究生从事 环境化学方面的研究工作, 1996到中国科学院长 春应用化学研究所读博士研究生从事分析化学方 面的研究工作, 2000年-2006年分别在瑞士苏黎世 联邦理工学院、美国俄克拉何马州立大学和密歇 根大学从事博士后研究, 开始专注于功能纳米材 料方面的研究。

问题2: 请介绍一下您的课题组在功能纳米材料领域 取得的主要成果?

回答: 我的课题组长期从事功能无机纳米粒子组 装体的设计、构筑、功能调控及应用研究。主要研 究成果包括: 1)组装基元的设计: 制备了形貌各向 异性的纳米粒子, 实现了对组装体成核和生长过 程的控制, 制备了宏观尺寸的 “类单晶” 三维有 序组装体; 2)组装基元间相互作用力的调控: 通过
调节纳米粒子的库仑排斥与范德华吸引力的平衡, 在溶液中实现了多种由多分散无机纳米粒子构建 的超级纳米粒子组装体的可控制备; 3)组装体的 构筑及应用: 构建了多种类、大尺寸、复杂结构、 具有特殊光学、电学、催化和机械性质的纳米粒子 组装体, 为纳米粒子材料在催化、能源等领域的最 终应用提供新的思路和理论依据。

问题 3: 请您简单回顾一下功能纳米材料领域最近 几年的重要进展?

回答: 最近几年功能纳米材料的发展非常迅速, 已 由早期的合成出不同尺寸大小和形貌的纳米材料 向 “精细化” 合成转变, 研究人员可以在原子和分 子水平的精准构筑纳米结构单元, 实现对其功能 的设计、剪裁和优化, 而且在能源、催化、生物和 环境等领域的应用不断出现新的研究成果。如纳 米催化方面提出了单原子/限域催化的概念, 生物 方面提出构筑智能响应型纳米机器人的概念, 能 源方面利用冷冻电镜观测电池材料和界面原子结 
构等等。

问题 4: 目前我国功能纳米材料研究在国际上处于 什么水平?

回答: 在国家的大力支持下, 我国纳米科技发展迅 速, 成果显著。在全方位、分层次的战略布局情况 下, 经过多年来长期的奋斗, 我国功能纳米材料领 域已形成了一支高水平的基础研究队伍, 取得了 一批国际上有影响的成果, 整体研究水平已进入 世界先进行列。以二维碳纳米材料的研究为例, 中 国科学院化学研究所李玉良老师原创性地提出了 石墨炔的概念, 在石墨烯的应用方面北京大学化 学与分子工程学院刘忠范老师的团队走在了世界 的最前列。

问题5: 功能纳米材料领域有迫切需要突破的卡脖 子技术吗？并分析功能纳米材料领域面临的一些 重要问题。

回答: 目前, 功能纳米材料的研究大都在实验室中 进行毫克级别制备, 产量比较小, 不能满足实际应 用的需求, 需要发展纳米材料的规模化制备技术, 提高其生产能力。然而, 采用一些放大的实验设备 进行合成时, 对应的反应环境会与微型反应器有 很大差异, 导致合成出的功能纳米材料的尺寸、形 貌、维度等可能会发生很大的变化, 进而导致其性 能发生明显的变化。因而, 功能纳米材料的规模化 制备技术成为限制其实际应用的一个瓶颈问题。

功能纳米材料领域面临的一些重要问题如 下: 1)如何实现微观和宏观的关联、物理化学性质 的跨尺度效应、非线性行为、多自由度耦合和调 控? 2)如何能够将纳米尺度的特性继续在宏观材 料中保持并得到优化? 3)如何使这些性能长时间 的发挥而不失效? 4)功能纳米材料的结构与性能 之间构效关系的研究不够深入, 缺乏一些原位的 表征技术。5)功能纳米材料的基础研究已经取得 了较高水平的成果, 但与企业的早期联系不够, 企 业的早期介入不够, 导致以应用为导向发展纳米 科技的力度不够, 与产业界脱离较远, 成果转化较 为缓慢。

问题 6: 功能纳米材料在哪些方面获得了实际应 用?

回答: 经过 20 多年的发展, 功能纳米材料在应用研 究方面取得了重要进展, 体现出纳米科技基础研 究成果对产业化发展的重大促进作用。如中国科
学院福建物质结构研究所与江苏丹化集团、上海 金煤化工新技术有限公司联手合作，成功开发了 煤制乙二醇系列纳米催化剂的研制与大化工技 术，该技术的推广应用将有效缓解我国乙二醇产 品供需矛盾; 中国科学院化学研究所研发的 “纳米 材料绿色印刷制版技术” 摒弃了感光成像的技术 思路, 从而有可能从根本上消除感光化学过程带 来的避光操作和废液排放问题; 国家纳米科学中 心、中国科学院过程工程研究所及中国电力科学 研究院合作完成的 “新型电力防污闪纳米复合室 温硫化硅橡胶(RTV)材料” 达到了国际领先水平, 该成果的完成是科研院所与用户单位共同攻克技 术难题, 基础研究和工程需求相结合的产物; 上海 硅酸盐研究所成功研制出针对汽车尾气净化、满 足严格国IV排放法规的新型高性能低成本 “三效” 催化剂, 为国产化高性能低成本汽车催化剂的研 发推广做出了贡献。

问题7: 您可否预测和分析一下功能纳米材料的现 状、发展方向?

回答: 随着合成化学的快速发展, 人们根据需求可 以设计和可控合成出具有不同尺寸、维度、化学组 分、形貌、功能的纳米材料, 已取得一系列的基础 研究成果, 而且在实际应用中也有了一些初步的 尝试。未来的发展需要集中纳米科技领域的优势 研究力量, 围绕功能纳米材料、纳米表征与标准、 纳米器件与制造、纳米催化、纳米生物与医学等重 点领域系统布局, 对有优势和引领作用的研究方 向强化支持, 解决纳米材料中若干重大共性和基 础性问题。面向能源、环境、健康等可持续发展领 域, 充分发挥功能纳米材料在经济、社会发展中的 引领作用, 从基础研究产生变革性技术, 带动和支 撑相关产业的发展。

问题 8: 您对功能纳米材料的学科发展有哪些期 望?

回答: 我对于功能纳米材料学科的发展有如下几 点期望：1)功能纳米材料研究逐步由主要集中在 基础研究逐渐向基础研究、应用研究及产业化并 举转变; 2) 由单一学科向多学科交叉和融合的方 向发展; 3)由独立工作向集成化和国际化方向发 展；4)更加重视关键装备的研发; 5)以材料为基础 向催化、能源、生物等方面的应用发展。

问题9: 您对准备从事功能纳米材料研究的青年学 者有何建议? 
回答: 我对准备从事功能纳米材料研究的青年学 者的建议如下: 1)选准研究方向。功能纳米材料是 一个涉及化学、物理、生物和材料科学多学科交叉 的前沿方向, 青年学者应该系统深入地了解该领 域的国际前沿, 掌握功能纳米材料精准构筑及其 应用中的重要进展、面临的难点和挑战性问题, 选 定自己感兴趣的研究方向。2)勤奋努力。青年学者 应该在自己的研究方向能够长期坚持不解的努 力, 耐得住寂寞, 保持与同行、学生间的交流, 提
升自己对研究领域中涉及的关键科学问题的更好 理解。3)不盲从热点。大家在了解国际前沿的发展 的时候, 能够客观评价相关方向的发展, 不要盲目 追寻科研中的热点方向, 保持科研之初心。4)瞄准 重大需求。从长远来看, 科研在推动科学不断发展 和完善的同时, 能否将其应用于实际生活, 把自己 的研究成果写作祖国的大地上, 服务于社会, 是大 家需要不断关注和思考的问题。

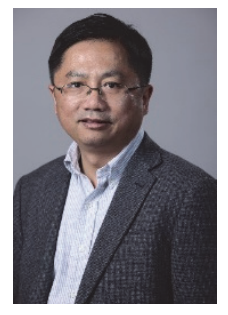

唐智勇, 国家纳米科学中心研究员, 中心副主任, 博士生导师。于1993和1996年在武 汉大学分别获得本科和硕士学位; 1999年在中国科学院长春应用化学研究所获得博士学 位; 2000至2006年分别在瑞士苏黎世联邦理工学院、美国俄克拉何马州立大学和密歇根大 学从事博士后研究; 2006年入选中国科学院 “百人计划”加入国家纳米科学中心。长期从 事功能无机纳米粒子组装体的设计、构筑、功能调控及应用研究。截止目前, 在自组装功 能纳米材料领域发表论文超250篇，包括以通讯作者发表Nature (1)、Nat. Energy (2)、Nat. Nanotechnol. (2)、Nat.Chem. (1)、Acc.Chem. Res. (1)、Chem. Soc. Rev. (3)、J. Am. Chem. Soc. (14)、Angew. Chem. Int. Ed. (18) (入选Author Profile)、Adv. Mater. (20) (入选Hall of Fame)、Nat. Commun. (2)、Sci. Adv. (3)等280余篇, 撰写中、英文专著各1部, 授权专利16件。截止2019年2月, 论文共被引用 25400 次, HIndex 为 83 , 入选科睿唯安化学、材料科学两个领域 “高被引科学家”、爱思唯尔材料科学 “中国高被引 学者”。2008年获中国科学院 “百人计划” 择优支持, 2010年获国家杰出青年科学基金资助, 2013年被 评为 “科技部中青年科技创新领军人才”, 2015年获北京市科学技术二等奖(排名第一), 2016年入选国家

“万人计划” 科技创新领军人才, 2018年获国家自然科学二等奖(排名第一), 获2019年发展中国家科学 院(TWAS)化学奖。 